\title{
Comparative analysis of EPA and DHA in fish oil nutritional capsules by GC-MS
}

\author{
Tao Yi ${ }^{*}$, Shuk-Man Li ${ }^{1}$, Jia-Yi Fan ${ }^{1}$, Lan-Lan Fan² ${ }^{2}$ Zhi-Feng Zhang ${ }^{3}$, Pei Luo ${ }^{3}$, Xiao-Jun Zhang ${ }^{4}$, Jian-Gang Wang ${ }^{4}$, \\ Lin Zhu', Zhong-Zhen Zhao ${ }^{1}$ and Hu-Biao Chen ${ }^{1 *}$
}

\begin{abstract}
Background: Fish oil is a popular nutritional product consumed in Hong Kong. Eicosapentaenoic acid (EPA) and docosahexaenoic acid (DHA) are the two main bioactive components responsible for the health benefits of fish oil. Market survey in Hong Kong demonstrated that various fish oil capsules with different origins and prices are sold simultaneously. However, these capsules are labelled with same ingredient levels, namely EPA $180 \mathrm{mg} / \mathrm{g}$ and DHA $120 \mathrm{mg} / \mathrm{g}$. This situation makes the consumers very confused. To evaluate the quality of various fish oil capsules, a comparative analysis of the contents of EPA and DHA in fish oil is crucial.
\end{abstract}

Methods: A gas chromatography-mass spectrometry (GC-MS) method was developed for identification and determination of EPA and DHA in fish oil capsules. A comprehensive validation of the developed method was conducted. Ten batches of fish oil capsules samples purchased from drugstores of Hong Kong were analyzed by using the developed method.

Results: The present method presented good sensitivity, precision and accuracy. The limits of detection (LOD) for EPA and DHA were $0.08 \mathrm{ng}$ and $0.21 \mathrm{ng}$, respectively. The relative standard deviation (RSD) values of EPA and DHA for repeatability tests were both less than 1.05\%; and the recovery for accuracy test of EPA and DHA were $100.50 \%$ and 103.83\%, respectively. In ten fish oil samples, the contents of EPA ranged from $39.52 \mathrm{mg} / \mathrm{g}$ to $509.16 \mathrm{mg} / \mathrm{g}$, and the contents of DHA ranged from $35.14 \mathrm{mg} / \mathrm{g}$ to $645.70 \mathrm{mg} / \mathrm{g}$.

Conclusion: The present method is suitable for the quantitative analysis of EPA and DHA in fish oil capsules. There is a significant variation in the contents of the quantified components in fish oil samples, and there is not a linear relationship between price and contents of EPA and DHA. Strict supervision of the labelling of the fish oil capsules is urgently needed.

Keywords: Fish oil, EPA, DHA, GC-MS, Comparative analysis

\section{Background}

Omega-3 polyunsaturated fatty acids, which include the fish oil components eicosapentaenoic acid (EPA) and docosahexaenoic acid (DHA, Figure 1), are essential for humans as cannot be synthesized by the human body [1]. It has been reported that omega-3 fatty acids are very important in preventing and managing heart disease [2]. Findings show omega-3 fish oil may help to lower blood pressure [3], reduce triglycerides accumulation [4], slow the development of plaque in the arteries [5], reduce the chance of abnormal heart rhythm [6], reduce the

\footnotetext{
* Correspondence: yitao@hkbu.edu.hk; hbchen@hkbu.edu.hk

${ }^{1}$ School of Chinese Medicine, Hong Kong Baptist University, Hong Kong

Special Administrative Region, Hong Kong, China

Full list of author information is available at the end of the article
}

likelihood of heart attack and stroke, and lessen the chance of sudden cardiac death in people with heart disease [7]. Omega-3 parenteral nutrition can reduce the rate of inflammatory complications after surgery [1]. It is also thought that EPA in particular may possess some beneficial therapeutic potential in mental conditions, such as schizophrenia, depression, hyperactivity and attention symptoms [8]. DHA is essential for the growth and functional development of the brain in infants [9], and is also required for maintenance of normal brain function in adults $[10,11]$. The FDA states it is safe to take up to $3 \mathrm{~g}$ of omega-3 per day to lower the risk for coronary heart disease (CHD) and maintain health [12]. Besides, the American Heart Association (AHA) recommends everyone eat fish (particularly fatty fish) at least twice a 


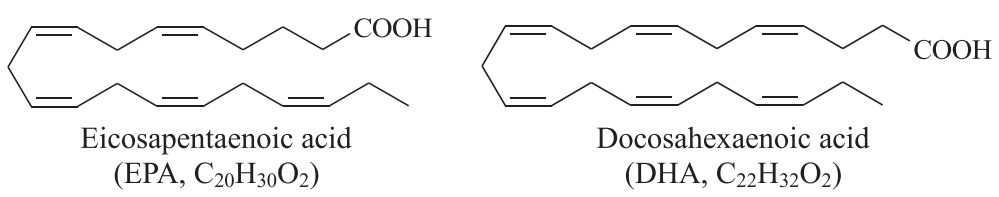

Figure 1 The chemical structures of EPA and DHA.

week. As omega-3 fatty acids are deemed important from authoritative bodies, supplementation in addition to food sources may need to be considered to help U.S. adults meet recommendations [13].

In the past 10 years, many people have taken omega- 3 fish oil supplements for their health benefits. Hong Kong is one of the strongest markets for health supplements in Asia [14], and more and more fish oil products are being sold in the markets. These fish oils come from different sources, and product prices vary significantly. However, these products are labelled with same ingredient levels, namely EPA $180 \mathrm{mg} / \mathrm{g}$ and DHA $120 \mathrm{mg} / \mathrm{g}$ (Table 1). Confronted with this variety, consumers are eager to know whether these fish oils contain same EPA and DHA contents? Whether there is relationship between the EPA/DHA contents and the price or source? Are the more expensive fish oil products really of better quality? To answer these questions, a comparative analysis of the contents of EPA and DHA in commercially available fish oil is urgently needed.

The determination of EPA and DHA in fish oil has been reported by high-performance liquid chromatography (HPLC) $[15,16]$ and liquid chromatography-mass spectrometry (LC-MS) $[17,18]$. However, the HPLC is not sensitive enough and the cost of LC-MS is high. In the present study, a new gas chromatography-mass spectrometry (GC-MS) method was developed for the analysis of fish oil. The samples bought from different drugstores in
Hong Kong were analyzed to determine DHA and EPA contents. The results demonstrated that our method is highly precise and accurate, and is therefore suitable for the determination of EPA and DHA in fish oil. Significant variation in the contents of EPA and DHA in fish oil samples was founded, and there is not a linear relationship between price and contents of EPA and DHA. Higher price can not guarantee higher contents of EPA and DHA. But because fish oil is expensive and its health claims are so significant, this is one product the government should seriously monitor.

\section{Experimental \\ Materials}

The sources of the fish oil capsule samples are listed in Table 1. Corresponding voucher specimens were deposited in the School of Chinese Medicine, Hong Kong Baptist University.

\section{Reagents and chemicals}

The standard compounds of eicosapentaenoic acid methyl ester, docosahexaenoic acid methyl ester and Supelco 37 component fame mix were purchased from Sigma-Aldrich (St. Louis, MO, USA). The purity of these chemical standards was more than $98 \%$ by GC-MS.

$N$-hexane was used as a solvent in GC-MS analysis, which was purchased from the RCI Lab-Scan Limited (Bangkok, Thailand). Potassium hydroxide and sodium

Table 1 The original sources, prices and contents of EPA and DHA in ten batches of fish oil capsule samples bought in Hong Kong $(n=3)$

\begin{tabular}{|c|c|c|c|c|c|c|}
\hline \multirow{2}{*}{$\begin{array}{c}\text { Sample } \\
\text { no. }\end{array}$} & \multirow{2}{*}{$\begin{array}{l}\text { Labelled } \\
\text { origin }\end{array}$} & \multirow{2}{*}{$\begin{array}{c}\text { Price } \\
(\mathrm{HK} \$ / \mathrm{g})\end{array}$} & \multicolumn{2}{|c|}{ Labelled contents (mg/g) } & \multicolumn{2}{|c|}{ Determined contents $(\mathrm{mg} / \mathrm{g})$} \\
\hline & & & EPA & DHA & EPA & DHA \\
\hline $1^{a}$ & USA & 1.98 & \multirow{10}{*}{180} & \multirow{10}{*}{120} & 200.45 & 201.45 \\
\hline 2 & Australia & 1.68 & & & 148.05 & 137.15 \\
\hline 3 & USA & 1.53 & & & 509.16 & 501.18 \\
\hline 4 & USA & 1.49 & & & 418.54 & 645.70 \\
\hline 5 & Australia & 1.02 & & & 132.44 & 123.26 \\
\hline 6 & USA & 0.98 & & & 361.36 & 419.73 \\
\hline 7 & New Zealand & 0.65 & & & 208.42 & 204.72 \\
\hline 8 & USA & 0.64 & & & 166.42 & 125.33 \\
\hline 9 & USA & 0.34 & & & 39.52 & 35.14 \\
\hline 10 & USA & 0.23 & & & 112.19 & 153.11 \\
\hline
\end{tabular}

a Samples are listed in order of decreasing price; that is, the most expensive are listed first. 

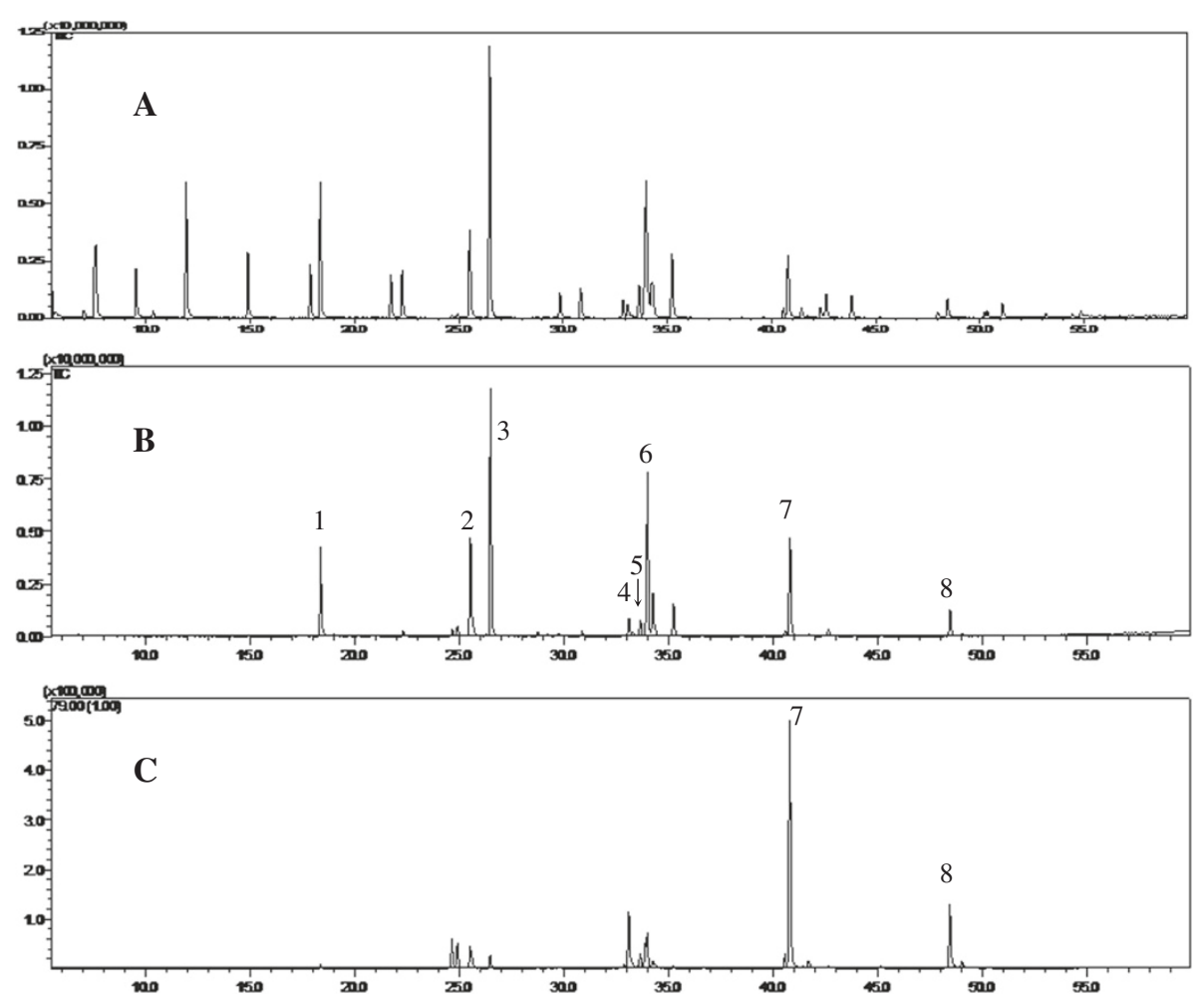

Figure 2 Typical GC-MS chromatograms of (A) the mix standard and (B) fish oil sample at full scan mode; and (C) fish oil sample at selective ion mode. 1, Methyl myristate (18.4 min); 2, Methyl palmitoleinate (25.5 min); 3, Methyl palmitate (26.5 min); 4, Methyl heptadecanoate (33.1 min); 5, Linoleic acid methyl ester (33.7 min); 6, Octadecenoic acid methyl ester (34.0 min); 7, Eicosapentaenoic acid methyl ester (40.8 min); 8, Docosahexaenoic acid methyl ester (48.5 min).

chloride of analytical grade was purchased from Uni-Chem (Shanghai, China). Boron trifluoride methanol complex solution (13-15\% $\mathrm{BF}_{3}$ basis), used to carry out methyl esterification, was purchased from Sigma-Aldrich (St. Louis, MO, USA). Water was purified using a Milli-Q water system (Millipore; Bedford, MA, USA).

\section{GC-MS instrumentation and conditions}

Shimadzu QP2010 GC-MS system (Kyoto, Japan) was used for qualitative and quantitative analysis of fish oil. DB-5 ms high resolution capillary column (Dikma Technologies. thickness: $0.25 \mu \mathrm{m}$, length: $30 \mathrm{~m}$, diameter: $0.25 \mathrm{~mm}$ ) was used for sample separation.

For temperature programming, the oven was maintained at $80^{\circ} \mathrm{C}$ for one minute and then increased at a rate of $10^{\circ} \mathrm{C}$ per minute to $250^{\circ} \mathrm{C}$, the rate was then slowed to $8^{\circ} \mathrm{C}$ per minute until $280^{\circ} \mathrm{C}$ was reached and maintained for $5 \mathrm{~min}$. Split injection was conducted with a split ratio of 10:1, and helium was used as the carrier gas at a rate of $0.8 \mathrm{ml} / \mathrm{min}$, with the volume of injection as $1 \mu \mathrm{L}$. The mass spectrometer was operated in electron-impact (EI) mode. Precolumn pressure: $70 \mathrm{kPa}$. Injection temperature: $250^{\circ} \mathrm{C}$. Ion source: $\mathrm{EI}\left(200^{\circ} \mathrm{C}\right)$. Interface temperature: $280^{\circ} \mathrm{C}$. Electron energy: $70 \mathrm{eV}$. Solvent delay: $5.5 \mathrm{~min}$. For qualitative analysis, the full scan mode was used and the scan range was $40-400 \mathrm{~m} / \mathrm{z}$. For quantitative analysis, selective ion mode was used, and $\mathrm{m} / \mathrm{z} 79$ was chosen as the ion fragment of EPA and DHA.

\section{Preparation of standard and sample solutions}

The stock solutions of EPA methyl ester $(5 \mathrm{mg} / \mathrm{L})$ and DHA methyl ester $(2.5 \mathrm{mg} / \mathrm{L})$ were prepared in $n$-hexane and stored in the refrigerator. The working solutions were prepared by appropriate dilution of the stock solutions with $n$-hexane, and the resulting concentrations of were 1 , $2.5,5,10,20,25$ and $30 \mathrm{mg} / \mathrm{L}$. DHA was prepared in serial dilutions of 2, 5, 10, 20, 40, 50 and $60 \mathrm{mg} / \mathrm{L}$. Calibration standard solution $(1 \mu \mathrm{L})$ was injected for GC-MS analysis.

The preparation of sample solutions was performed as previously described with modifications [19]. Samples were obtained from fish oil capsules by puncturing the capsule with a needle syringe. Each sample of approximately $60 \mathrm{mg}$ was weighed accurately and placed in a centrifuge tube with a ground stopper. $3 \mathrm{~mL}$ potassium hydroxide methanol solution $(0.5 \mathrm{M})$ was added. The contents were thoroughly mixed, and then the tube was filled with nitrogen gas, heated in a water bath at $60^{\circ} \mathrm{C}$, shaken three times in the course of $20 \mathrm{~min}$. When the oil droplets had disappeared completely and the solution was transparent, $3 \mathrm{~mL}$ boron trifluoride methanol complex solution was added, 
and the mixture cooled. Each tube was then filled with nitrogen gas, and placed in a water bath at $60^{\circ} \mathrm{C}$ for $5 \mathrm{~min}$. Saturated sodium chloride solution of $2 \mathrm{~mL}$ and $n$-hexane of $2 \mathrm{~mL}$ were added and mixed well. After centrifugation (4000 rpm) for $10 \mathrm{~min}$, the supernatant was drawn off, to be used as sample solution. Dilution of the supernatant is necessary in case its concentration falls out of the linear range. An aliquot of $1 \mu \mathrm{L}$ supernatant was injected for GC-MS analysis.

\section{Assay validation and sample determination}

Linearity for standards was determined with five data points over the concentration range of the working solutions. Precision was evaluated by six injections of the sample solution (batch 1) within one day. Repeatability was evaluated in intra- and inter-day assays of the fish oil sample FO1. The stability test was performed by analyzing the sample solution (batch 1 ) over a period of $24 \mathrm{~h}$. The relative standard deviation (RSD) was taken as the measures of precision, repeatability and stability. Recovery of all the quantified constituents was determined by sample in different concentration levels using a mixture of standards with 50, 100 and $200 \%$ of the quantified levels of constituents in the samples. All fish oil samples were analyzed using this method, and the acid/ester conversion factor was set to 0.96 .

\section{Results and discussion}

Optimization of hydrolysis and esterification conditions

EPA and DHA are present in fish oil in the form of various triglycerides. To generate a volatile DHA methyl ester for GC-MS analysis, EPA and DHA are released from the triglycerides by hydrolysis, and then methyl esterified. Various hydrolysis and methyl esterification conditions (different time, temperature, usage of nitrogen gas) were evaluated to obtain maximum extraction efficiency. The results demonstrated that incubation of fish oil (c.a. $60 \mathrm{mg})$ in potassium hydroxide methanol solution $(0.5 \mathrm{M}$, $3 \mathrm{~mL}$ ) at $60^{\circ} \mathrm{C}$ for 20 min achieved complete hydrolysis. Esterification with $3 \mathrm{~mL}$ boron trifluoride methanol solution at $60^{\circ} \mathrm{C}$ for 5 min provides the most methyl esters. Moreover, nitrogen used as protection gas ensures less oxidation and higher stability of the constituent contents. After further optimization, the best experimental conditions are shown in "Preparation of standard and sample solutions".

\section{Chromatographic conditions and GC-MS identification}

The chromatographic conditions such as temperature gradient and carrier gas flow rate were optimized to achieve satisfactory separation and sharp peak shape in the chromatograms. Full scan mode was used in qualitative tests because it provides more peaks for identification. For the qualitative analysis, apart from EPA (7) and DHA
Table 2 Linearity calibration curve factors, LOD and LOQ of EPA and DHA

\begin{tabular}{cccccc}
\hline Analyte & Equation & $\begin{array}{c}\text { Range } \\
(\mathbf{m g} / \mathbf{L})\end{array}$ & $\boldsymbol{R}^{\mathbf{2}}$ & LOD (ng) & LOQ (ng) \\
\hline EPA & $y=41328.56 x-7.85$ & $1-30$ & 0.9999 & 0.08 & 0.15 \\
DHA & $y=11863.62 x+4.62$ & $2-60$ & 0.9999 & 0.21 & 0.60 \\
\hline
\end{tabular}

(8), 6 characteristic peaks of constituent chemicals had been successfully identified with the aid of the reference standard. They are methyl myristate (1), methyl palmitoleinate (2), methyl palmitate (3), methyl heptadecanoate (4), linoleic acid methyl ester (5), and octadecenoic acid methyl ester (6). The typical GC-MS chromatograms are shown in Figure 2A and 2B.

In quantitative test, selective ion mode (SIM) was used due to its higher sensitivity. Considering the abundance of fragment ions of EPA and DHA in mass spectra (Additional file 1: Figure S1), m/z 79 was used for calculating amount of EPA and DHA. The typical chromatogram is shown in Figure 2C.

\section{Validation of the analysis method}

Results for assessment of the validity of the method are summarized in Table 2, 3 and 4. The data indicate good linearity between concentrations and peak areas of the analytes within the test ranges. The limits of detection (LOD) for EPA and DHA were found to be $0.08 \mathrm{ng}$ and $0.21 \mathrm{ng}$, respectively. Therefore, the system was considered to be sensitive. The relative standard deviation (RSD) values of intra-day and inter-day variations were not more than $0.59 \%$ and $1.00 \%$ for EPA, and not more than $1.08 \%$ and $1.05 \%$ for DHA, respectively. The established method also had acceptable accuracy with average recovery of $100.50 \%$ and $103.83 \%$ for EPA and DHA. All these results demonstrate that the developed GC-MS method was sufficiently reliable and accurate and is therefore suitable for quantification of EPA and DHA in fish oil.

\section{Sample analysis}

The present method was successfully applied to the quantification of EPA and DHA in fish oil capsule samples, and the results are summarized in Table 1 and Figure 3. The results reveal significant variation in the contents of the quantified components in fish oil samples. Such variations

Table 3 Precision, repeatability and stability of EPA and DHA

\begin{tabular}{|c|c|c|c|c|c|c|}
\hline \multirow[t]{2}{*}{ Analyte } & \multirow[t]{2}{*}{$\begin{array}{c}\text { Precision } \\
\text { (RSD, \%, } n=3)\end{array}$} & \multicolumn{4}{|c|}{$\begin{array}{c}\text { Repeatability } \\
\text { (RSD, \%, } n=3 \text { ) }\end{array}$} & \multirow[t]{2}{*}{$\begin{array}{c}\text { Stability } \\
(\mathrm{RSD}, \%, n=6)\end{array}$} \\
\hline & & $\begin{array}{c}\text { Day } \\
1\end{array}$ & $\begin{array}{c}\text { Day } \\
2\end{array}$ & $\begin{array}{c}\text { Day } \\
3\end{array}$ & Inter-day & \\
\hline EPA & 0.38 & 0.38 & 0.59 & 0.16 & 1.00 & 0.85 \\
\hline DHA & 0.32 & 0.08 & 0.32 & 1.08 & 1.05 & 0.63 \\
\hline
\end{tabular}


Table 4 Recovery of EPA and DHA

\begin{tabular}{|c|c|c|c|c|c|c|c|c|}
\hline \multirow[t]{2}{*}{ Analyte } & \multicolumn{2}{|c|}{$50 \%$} & \multicolumn{2}{|c|}{$100 \%$} & \multicolumn{2}{|c|}{$200 \%$} & \multicolumn{2}{|c|}{ Average } \\
\hline & Recovery (\%) & RSD (\%) & Recovery (\%) & RSD (\%) & Recovery (\%) & RSD (\%) & Recovery (\%) & RSD (\%) \\
\hline EPA & $104.10 \pm 3.71^{a}$ & 3.56 & $99.85 \pm 2.15$ & 2.15 & $97.55 \pm 0.80$ & 0.82 & $100.50 \pm 3.32$ & 3.31 \\
\hline DHA & $104.70 \pm 1.94$ & 1.85 & $101.22 \pm 2.38$ & 2.35 & $105.56 \pm 2.42$ & 2.29 & $103.83 \pm 2.30$ & 2.21 \\
\hline
\end{tabular}

Values shown are mean \pm SD $(n=3)$.

may be mainly due to the source and processing of the fish oil.

Fish oils are commercially produced from cold water fatty fish, including salmon, tuna, sardines, shellfish, and herring. The contents of EPA and DHA in theses fishes are significantly varied [20,21]. During the filling of soft capsules, poor quality fish oil or even vegetable oil may be added thereby adulterating good quality fish oil [22]. Adulteration can induce unstable contents of EPA and DHA in the final products, which make the actual contents do not match the label.

Figure 3 also shows that the relationship between the price and the contents of DHA and EPA are insignificant. In other words, according to the prices for the various fish oil sold in markets, it was clear that the contents of EPA and DHA did not always correlate with price. For example, sample 3, 4 and 6 contained higher contents of EPA and DHA but it was cheaper than samples 1, 2 and 5 . Distinctly, the classification of various prices of fish oil actually did not distinguish relative quality. This finding confirms the need for developing a reliable evaluation method to ensure the quality of fish oil products.

\section{Conclusions}

A GC-MS method was developed for determination of EPA and DHA content in fish oil capsules. A comprehensive evaluation of the developed method was conducted, and the method was shown to be highly sensitive, reproducible and accurate. Samples of 10 commercial fish oil capsule samples bought in Hong Kong retail stores were tested. The results demonstrated significant variation in

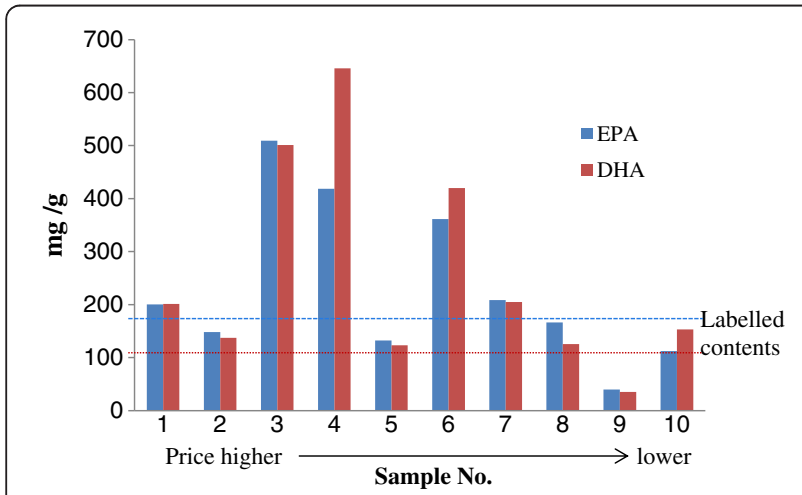

Figure 3 The contents of EPA and DHA in ten batches of fish oil capsule samples as compared to price $(n=3)$. the contents of EPA and DHA in the samples, and there is not a linear relationship between price and contents of EPA and DHA. Strict supervision of the labelling of the fish oil capsules is urgently needed.

\section{Additional file}

Additional file 1: Figure S1. The mass spectra of (a) EPA and (b) DHA methyl ester.

\section{Abbreviations}

EPA: Eicosapentaenoic acid; DHA: Docosahexaenoic acid; GC-MS: Gas chromatography-mass spectrometry; LOD: Limit of detection; LOQ: Limit of quantitation.

\section{Competing interests}

The authors declare that they have no competing interests.

\section{Authors' contributions}

TY and HBC initiated the study; all authors contributed to designing the study. The sample extraction was conduct by SML, JYF and LZ. The optimization of experimental conditions was performed by LLF, ZFZ and PL. The data analysis was conduct by XJZ, JGW and ZZZ. TY and SML drafted the manuscript. All authors contributed to data analysis and manuscript finalization. All authors read and approved the final manuscript.

\section{Acknowledgements}

This work was financially supported by the National Science Fund (no.31060055) of the People's Republic of China.

\section{Author details}

'School of Chinese Medicine, Hong Kong Baptist University, Hong Kong Special Administrative Region, Hong Kong, China. ${ }^{2}$ Guangxi Botanical Garden of Medicinal Plant, Nanning, Guangxi 530023, China. ${ }^{3}$ The State Key Laboratory of Quality Research in Chinese Medicine, Macau University of Science and Technology, Macau, China. ${ }^{4}$ School of Chinese Medicine, Guangzhou University of Traditional Chinese Medicine, Guangzhou, China.

Received: 7 July 2014 Accepted: 9 December 2014

Published: 13 December 2014

\section{References}

1. Wei Z, Wang W, Chen J, Yang D, Yan R, Cai Q: A prospective, randomized, controlled study of $\omega$-3 fish oil fat emulsion-based parenteral nutrition for patients following surgical resection of gastric tumors. Nutr J 2014, 13:25.

2. Kris-Etherton PM, Harris WS, Appel L: Fish consumption, fish oil, omega-3 fatty acids and cardiovascular disease. Circulation 2002, 106:2747-2757.

3. Woodman RJ, Mori TA, Burke V, Puddey IB, Watts GF, Beilin LJ: Effects of purified eicosapentaenoic and docosahexaenoic acids on glycemic control, blood pressure, and serum lipids in type 2 diabetic patients with treated hypertension. Am J Clin Nutr 2002, 76:1007-1015.

4. Beynen AC, Katan MB: Why do polyunsaturated fatty acids lower serum cholesterol? Am J Clin Nutr 1985, 42:560-563.

5. Connor SL, Connor WE: Are fish oils beneficial in the prevention and treatment of coronary artery disease? Am J Clin Nutr 1997, 66:1020S-1031S.

6. Breslow JL: n-3 Fatty acids and cardiovascular disease. Am J Clin Nutr 2006, 83:S1477-1482S. 
7. Delgado-Lista J, Perez-Martinez P, Lopez-Miranda J, Perez-Jimenez F: Long chain omega-3 fatty acids and cardiovascular disease: a systematic review. Br J Nutr 2012, 107:S201-S213.

8. Féart C, Peuchant E, Letenneur L, Samieri C, Montagnier D, Fourrier-Reglat A, Barberger-Gateau P: Plasma eicosapentaenoic acid is inversely associated with severity of depressive symptomatology in the elderly: data from the Bordeaux sample of the Three-City Study. Am J Clin Nutr 2008, 87:1156-1162.

9. Rizzo AM, Corsetto PA, Montorfano G, Opizzi A, Faliva M, Giacosa A, Ricevuti G, Pelucchi C, Berra B, Rondanelli M: Comparison between the AA/EPA ratio in depressed and non depressed elderly females: omega-3 fatty acid supplementation correlates with improved symptoms but does not change immunological parameters. Nutr J 2012, 11:82.

10. Innis SM: Dietary (n-3) fatty acids and brain development. J Nutr 2007, 137:855-859.

11. Stonehouse W, Conlon CA, Podd J, Hill SR, Minihane AM, Haskell C, Kennedy D; DHA supplementation improved both memory and reaction time in healthy young adults: a randomized controlled trial. Am J Clin Nutr 2013, 97:1134-1143.

12. Letter Responding to Health Claim Petition dated June 23, 2003 (Wellness petition): Omega-3 Fatty Acids and Reduced Risk of Coronary Heart Disease (Docket No. 2003Q-0401) http://www.fda.gov/Food/

IngredientsPackagingLabeling/LabelingNutrition/ucm072936.htm.

13. Papanikolaou Y, Brooks J, Reider C, Fulgoni VL: U.S. adults are not meeting recommended levels for fish and omega-3 fatty acid intake: results of an analysis using observational data from NHANES 2003-2008. Nutr J 2014, 13:31.

14. Tsi D, Poon WM: Hong Kong regulatory overview for health supplements, EAS Strategic Advice Pte Ltd, Engredea News \& Analysis; 2009.

15. Teng Jl, Gowda NMM: Analysis of $n-3$ fatty acids in fish oils by highperformance liquid chromatography. Chromatographia 1993, 35:627-630

16. Batta AK, Dayal V, Colman RW, Shinha AK: Separation of underivatized $C_{20}$ fatty acids by reversed-phase high-performance liquid chromatography. J Chromatogr A 1984, 284:257-260.

17. Lacaze JP, Stobo LA, Turrell EA, Quilliam MA: Solid-phase extraction and liquid chromatography-mass spectrometry for the determination of free fatty acids in shellfish. J Chromatogr A 2007, 1145:51-57.

18. Salm P, Taylor PJ, Kostner K: Simultaneous quantification of total eicosapentaenoic acid, docosahexaenoic acid and arachidonic acid in plasma by high-performance liquid chromatography-tandem mass spectrometry. Biomed Chromatogr 2011, 25:652-659.

19. Li B, Yang JX, Han L, Guo DH, Xiong LB: A gas chromatography and mass spectrum study of unsaturated fatty acids in fish oil. Sh J Prev Med 2004, 16:211-214.

20. Teale MC: Omega 3 fatty acid research. New York: Nova; c2006.

21. Falk-Petersen S, Sargent JR, Henderson J, Hegseth EN, Hop H, Okolodkov YB: Lipids and fatty acids in ice algae and phytoplankton from the marginal ice zone in the Barents Sea. Polar Biol 1998, 20:41-47.

22. Pizzorno JE, Murray MT: Textbook of Natural Medicine. 4th edition. London: Churchill Livingstone; 2012.

doi:10.1186/1476-511X-13-190

Cite this article as: Yi et al.: Comparative analysis of EPA and DHA in fish oil nutritional capsules by GC-MS. Lipids in Health and Disease 2014 13:190,

\section{Submit your next manuscript to BioMed Central and take full advantage of:}

- Convenient online submission

- Thorough peer review

- No space constraints or color figure charges

- Immediate publication on acceptance

- Inclusion in PubMed, CAS, Scopus and Google Scholar

- Research which is freely available for redistribution 